\title{
Study of Dynamics of Block-Media in the Framework of Minimalistic Numerical Models
}

\author{
Alexander E. Filippov and Valentin L. Popov
}

\begin{abstract}
One of the principal methods of preventing large earthquakes is stimulation of a large series of small events. The result is a transfer of the rapid tectonic dynamics in a creep mode. In this chapter, we discuss possibilities for such a transfer in the framework of simplified models of a subduction zone. The proposed model describes well the basic characteristic features of geo-medium behavior, in particular, statistics of earthquakes (Gutenberg Richter and Omori laws). Its analysis shows that local relatively low-energy impacts can switch block dynamics from stick-slip to creep mode. Thus, it is possible to change the statistics of seismic energy release by means of a series of local, periodic, and relatively low energy impacts. This means a principal possibility of "suppressing" strong earthquakes. Additionally, a modified version of the Burridge-Knopoff model including a simple model for state dependent friction force is derived and studied. The friction model describes a velocity weakening of friction between moving blocks and an increase of static friction during stick periods. It provides a simplified but qualitatively correct stability diagram for the transition from smooth sliding to a stick-slip behavior as observed in various tribological systems. Attractor properties of the model dynamic equations were studied under a broad range of parameters for one- and two-dimensional systems.
\end{abstract}

Keywords Earthquakes · Block-media motion - Numerical simulation • Burridge-knopoff model $\cdot$ Stick-slip $\cdot$ Phase diagram $\cdot$ Seismic shocks $\cdot$ Phase transition - Time dependent friction

\footnotetext{
A. E. Filippov ( $\varangle)$

Donetsk Institute for Physics and Engineering, NASU, Donetsk 83114, Ukraine

e-mail: filippov_ae@yahoo.com

V. L. Popov

Technische Universität Berlin, 10623 Berlin, Germany

e-mail:v.popov@tu-berlin.de 


\section{Introduction}

An important and interesting application of studies of spatial-temporal pattern formation of mechanical systems is the formation of geological faults and their dynamics. Of particular interest is the study of the possibility of changing the mode of the fault dynamics into a slow one, thus preventing strong seismic shocks. The fact that the statistics of earthquake magnitude and their time correlations meet the laws of Gutenberg Richter [1, 2] and Omori [1, 3], typical for self-organized critical systems $[4,5]$, is often used for the conclusion that it principally occurs on all spatial scales ranging from microscopic to continental plate scale. Therefore, it is impossible to exert a targeted influence on the dynamics of earthquakes by local effects of limited energy. However, the works [6-10], based both on modeling by movable cellular automaton and full-scale experiments (on one of the active faults of the Baikal rift zone) suggest the principal possibility of releasing the accumulated elastic energy due to controlled low energy actions (vibration load and watering).

Based on this idea, we develop and study a model of the behavior of contact zones of block media and analyze the possibility of controlling the mode of displacement as was found experimentally.

\section{Mechanical Model}

Minimalistic mechanical model demonstrating the time correlations typical for systems showing self-organized criticality has been suggested by the authors. In the conceptual form, it is shown in Fig. 1. The plate is moved by the external force of $F_{\text {ext }}$. The plate is inclined by an angle that determines the ratio between the vertical component of the force $F_{\text {ext }} \cos (\alpha)$ acting against the force of Archimedes, which supports the plate "in magma", and the horizontal component, which results in the

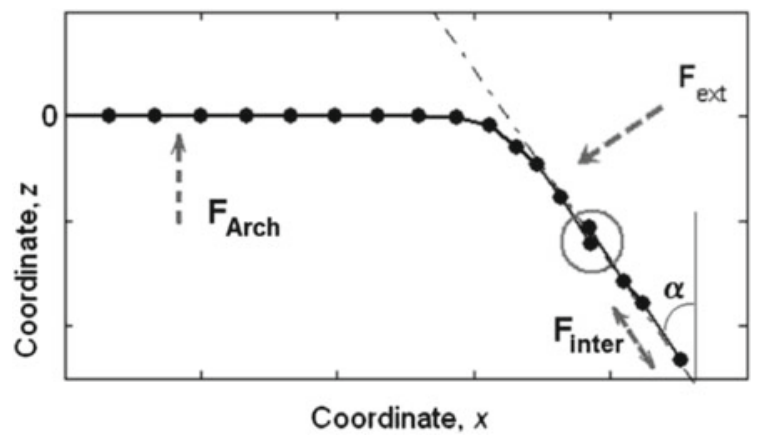

Fig. 1 Schematic diagram of the mechanical model. The force exerted by the mainland, $F_{\text {ext }}$, the force of Archimedes $F_{\text {Arch }}$ and the force of elastic interaction $F_{\text {inter }}$ are indicated. The circle shows the event of "bark fracture" 
displacement of the entire system along the $x$-axis and deformation of the elastic plate.

In a numerical simulation, the plate is transformed into a set of discrete elements connected by a (nonlinear) elastic force that tries to maintain a fixed distance between them. Let us first consider the simplest, two-dimensional version of the problem. In this case, the plate is transformed into an elastic chain, and the model equations are reduced to the following form:

$$
\begin{aligned}
& \partial x / \partial t=F_{\text {inter, } x}+F_{x}+\xi_{x} ; \\
& \partial z / \partial t=F_{\text {inter, } z}+F_{\text {Arh, } z}+F_{z}+\varepsilon_{z} ; \\
& \partial X / \partial t=\sum_{j} F_{x}(j)+F_{\text {ext }, x}+\varepsilon .
\end{aligned}
$$

Here, $F_{x}=F_{\text {ext }} \sin (\alpha)$ and $F_{z}=F_{\text {ext }} \cos (\alpha)$ are the projections of the force of the chain's interaction with the "mainland plate" (we neglect the vertical movement of the heavy mainland plate here), and the summation is made over all elements of the chain. The external force is assumed to be constant (acting on the drifting continent from the magma side). The levitation force of Archimedes $F_{\mathrm{Arch}, z}$ is given by a condition:

$$
F_{\text {Arch, } z}=\text { const } \cdot U_{0}>0 \text { at } z<0 \text { and } F_{\text {Arch, } z}=0 \text { at } z>0
$$

and $F_{\text {inter, } x}$ and $F_{\text {inter, } \mathrm{z}}$, components of nonlinear elasticity between the segments of the chain, are equal:

$$
F_{\text {inter, } x}=-\partial U_{\text {inter }} / \partial x \text { and } F_{\text {inter, } z}=-\partial U_{\text {inter }} / \partial z,
$$

where the distance-dependent effective interaction potential $U_{\text {inter }}$ looks like:

$$
U_{\text {inter }}(r)=K r^{2}\left(1-r^{2} / r_{0}^{2}\right) \text {. }
$$

Here, $K$ is the elastic constant. To fix the distance between the elements, in the simplest, most widespread approach [11-14], the potential of the fourth order (4) is used, for which the components of the forces between the elements contain cubic nonlinearity, providing the required rigidity. At the same time, in 2D (or 3D) space, the chain (surface) can bend under the influence of the force $F_{\text {ext }}$ and its elements move in the vertical (and/or horizontal) direction.

In numerical modeling, this leads to the following fracture condition. If the $x$ - or $z$ projection of the vector connecting two consecutive segments of the chain is negative and its absolute value exceeds some threshold value, then the "fracture" occurs in this place. The fragment of the chain from its beginning to this point is subsequently removed. In the absence of resistance on the part of the removed fragment, the speed of the continental plate sharply increases, up to its deceleration by further segments of 
the chain. Random influences from the surrounding subsystems are included through the $\delta$-correlated noise source:

$$
\begin{aligned}
\left\langle\xi_{x z}(t, x, z) \xi_{x z}\left(t^{\prime}, x^{\prime}, z^{\prime}\right)\right\rangle & =D_{1} \delta_{x z} \delta\left(t-t^{\prime}\right) \delta\left(x-x^{\prime}\right) \delta\left(z-z^{\prime}\right) \\
\left\langle\xi(t, x, z) \xi\left(t^{\prime}, x^{\prime}, z^{\prime}\right)\right\rangle & =D_{2} \delta\left(t-t^{\prime}\right) \delta\left(x-x^{\prime}\right) \delta\left(z-z^{\prime}\right) .
\end{aligned}
$$

Here $\delta(\ldots)$ is the impulse function of Dirac, $\delta_{x z}$ is the symbol of Kronnecker, and in each case some effective temperature can be assigned to the "diffusion coefficient" $D_{1,2}=2 k_{B} T_{1,2}$. The dissipative constant can be selected arbitrarily. It sets a characteristic time scale, and should be fitted a posteriori by experimental data. Random influences on the heavy mainland plate can be neglected, which leads to the assumption $D_{1} \gg D_{2}$.

\section{Statistical Properties of the Model}

At a constant external force $F_{\text {ext }}$, the movement occurs with a constant-on the large time scales - average velocity $\langle V\rangle=d X / d t$. If we subtract $V t$ from the $X(t)$ curve, the fine structure of the derivative $d X / d t$ becomes clearly visible when the chain breaks (see Fig. 2). The right side of Fig. 2 shows the distribution of the lengths of "jumps" and intervals between them.

Let us compare these distributions at different noise intensities. With exception of the expected reduction in the average interval between the jumps, the results do weakly dependend on the noise intensity up to the values comparable to the fracture threshold. Moderate noise (which does not exceed the dynamic chaos intensity of this
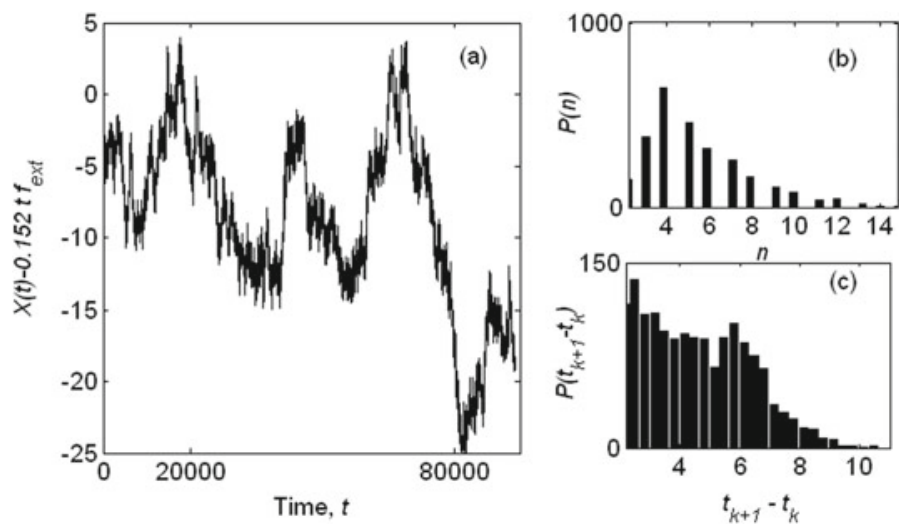

Fig. 2 The fine structure of the jumps a obtained by subtracting $V t$ and typical distributions of lengths of the chain fragments removed after fracture events (b) intervals between the jumps (c) obtained for parameter values $\alpha=\pi / 6, F_{\text {ext }}=10, U_{0}=0.1, K=0.1+0.9 \xi$ 
nonlinear system) only increases standard deviations. Therefore, we present below the results obtained at negligibly low noise.

Of interest is an analysis of the influence of regular (periodic) spatially localized actions on the system. We simulated the influence of sinusoidal and impulse actions of different intensity. Note that exactly the latter type of action is used in field experiments.

In the presence of noise, such actions reduce the average time between jumps. However, being spatially localized, they fix quite precisely both the time and length of each jump. This can be achieved by selecting resonant frequencies, amplitudes and force application points. Weak impact plays here the role of the trigger mechanism, provoking its own, more powerful processes in the system.

Formally, the model is able to achieve an accurate resonance optimum such that the random components are practically suppressed. In field conditions, the parameters are not as controlled as in the numerical experiment, and the histogram of jump distribution acquires additional lines near the resonant one.

\section{Three-Dimensional System and Reduced Frontal Motion Model}

Real tectonic systems are three-dimensional. Therefore, even for a minimalistic model, in addition to coordinates $(x, z)$ also the $y$-coordinate along the edge of the fault has to be considered. In a self-consistent approximation, we can assume that the $3 D$ system is composed of many equivalent $2 D$ systems, which interact only through a common front of contact with the "continent". All system Eq. (1) acquire an additional index [layer number $(x, z)$ ] along the $y$-axis

$$
\begin{aligned}
\partial x(k) / \partial t & =F_{\text {inter, } x}(k)+F_{x}(k)+\xi_{x}(k), \\
\partial z(k) / \partial t & =F_{\text {inter, } z}(k)+F_{\text {Arh, } z}(k)+F_{z}(k)+\xi_{z}(k) .
\end{aligned}
$$

The last equation of the system (1) is modified into equation:

$$
\partial X(k) / \partial t=\sum_{j} F_{x}(k)(j)+F_{\text {ext }, x}(k)+K[X(k+1)+X(k-1)-2 X(k)] .
$$

At small deviations between the neighboring layers, they behave quasi-independently according to the $2 D$ model described above. If the deviations between $X(k+1)+$ $X(k-1)$ and $2 X(k)$ increase, they are suppressed by the elastic bond $K[X(k+1)+$ $X(k-1)-2 X(k)]$ determined by the constant $K$.

In the following, we will shift each following action along the front by some value so that at the present moment the small jump will be (with certain probability) provoked in another place of the front. Figure 3 illustrates the resulting propagation 


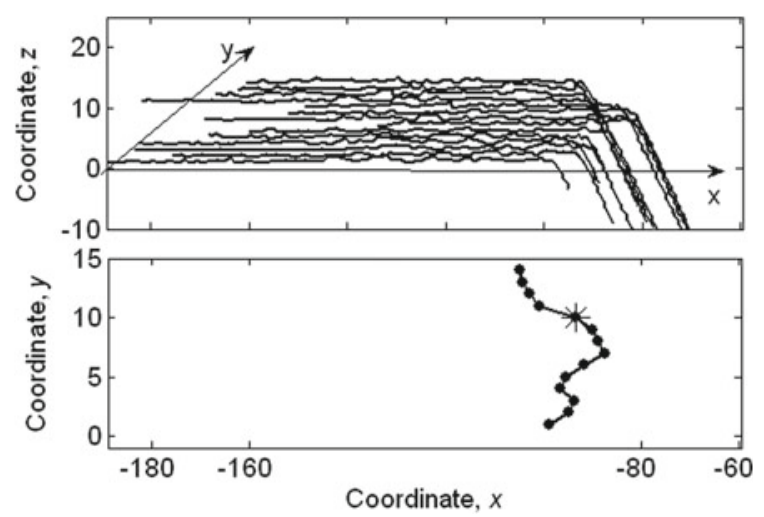

Fig. 3 The movement of the frontal area of $X(k)$ caused by periodic impacts along it

of the front on example fraction of the front. Moving the impact point along the front (asterisk in Fig. 3) generates multiple front jumps (instead of one), thus facilitating small jumps. The corresponding histograms contain some contribution of jumps, large in amplitude and time intervals between them, which, however, is much smaller than for an unperturbed system.

Taking into account the practical importance of such a problem, as well as the general scientific interest, it is useful to construct a simplified minimalist model of frontal motion, in which the connection between the layers would be taken into account in the rules of advancement of its fragments. For this purpose, let us consider a $2 D$ front line in the plane $(x, y)$, each segment of which moves forward under the action of a constant external force.

Such a minimalistic model successfully reproduces the basic properties of a more general $3 D$-model, and is compact enough for large parameter studies. It is stable against varying model parameters in a very wide range. First, we checked that the change of the elastic constant by three orders of magnitude did not led to any substantial change in the distribution function $P[y(k)]$.

The method of inducing local surges described above works with the reduced model in the same manner as described above in a 3D model. In other words, it is possible to select such periodicity and distance between the local impacts that they provoke a wave of small jumps, which leads to an almost regular movement of the entire front line. The instantaneous state of this process is shown in Fig. 4. For the sake of clarity, the planar front has been chosen here as the initial condition. The area near the artificial influence is marked with a grey circle. The instantaneous position of the impact zone is shown by a dark solid circle; the spontaneous jumps caused by it are marked with bold dots inside the circle.

The numerical model above, in its minimalistic variant, was published by the authors [12] in 2006 and was used to study the dynamics of subduction zone dynamics. Later on, in 2008, the detected correlations were used by us [13] to select the optimal scenarios of other "weak and cheap" local energy effects. As a result, the 

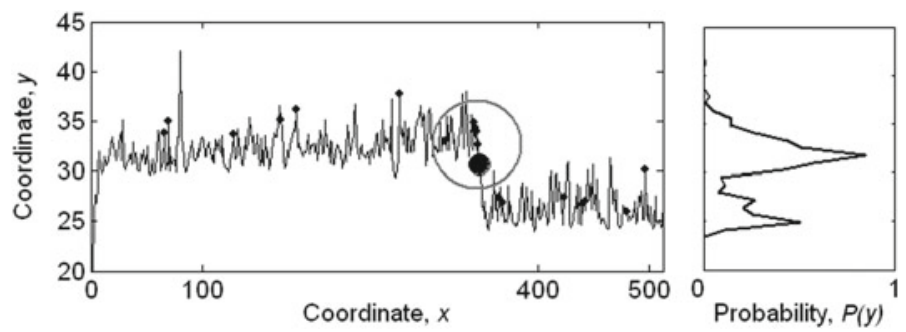

Fig. 4 The initial stage of the formation of an equilibrium distribution of probability $P[y(k)]$ in the minimalist model at the $2 \mathrm{D}$ front

efficiency of such impacts was significantly increased making it possible to switch the movement of blocks between "stick-slip" and creep modes. The average energy of single seismic shocks was significantly reduced and it became possible to "suppress" strong earthquakes. The model adequately described the laws of deformation for a block system and the temporal correlations typical of systems with self-organized criticality. The proposed model differs in principle from those studied previously (see, e.g., [2-11]) by taking into account the real topology of the creep of a continental platform on a thinner oceanic platform.

\section{Correlation Functions}

The simplicity and numerical efficiency of the model described in the previous Sections can be used to accumulate the statistics of correlations of the motion of separate blocks at the front. These correlations, in turn, can be used for selecting an optimum scheme of external action leading to the transformation of the system dynamics into a creep regime. Consider a $2 D$ front in the $(x, y)$ plane, each segment of which is moved forward by an over damped external force. Let us assume that every subsequent "tectonic" jump of each segment takes place upon its displacement by a certain distance, which is generated by a random number generator $\left\langle\xi(t, x, z) \xi\left(t^{\prime}, x^{\prime}, z^{\prime}\right)\right\rangle=D_{2} \delta\left(t-t^{\prime}\right) \delta\left(x-x^{\prime}\right) \delta\left(z-z^{\prime}\right)$ with zero mean value $\langle\xi(t, x, z)\rangle=0$.

The jump magnitude $\psi$ is also assumed to be a random quantity with zero mean value $\langle\psi(k, t)\rangle=0$ and is set by the average weak noise intensity $D_{\psi} \ll D_{\xi}$ as follows:

$$
\left\langle\psi(k, t) \psi\left(k^{\prime}, t^{\prime}\right)\right\rangle=D_{\psi} \delta_{k k^{\prime}} \delta\left(t-t^{\prime}\right)
$$

The particular noise intensity $D_{\psi}$ has to be reconstructed a posteriori from experimental data. Following article [12], we assumed that the coordinate $X(k)$ depends on the number of layers $k$ and that different layers are coupled by the elastic force $F_{\text {elastic }}=K[X(k+1)+X(k-1)-2 X(k)]$ already used in Eq. (7). Other details 


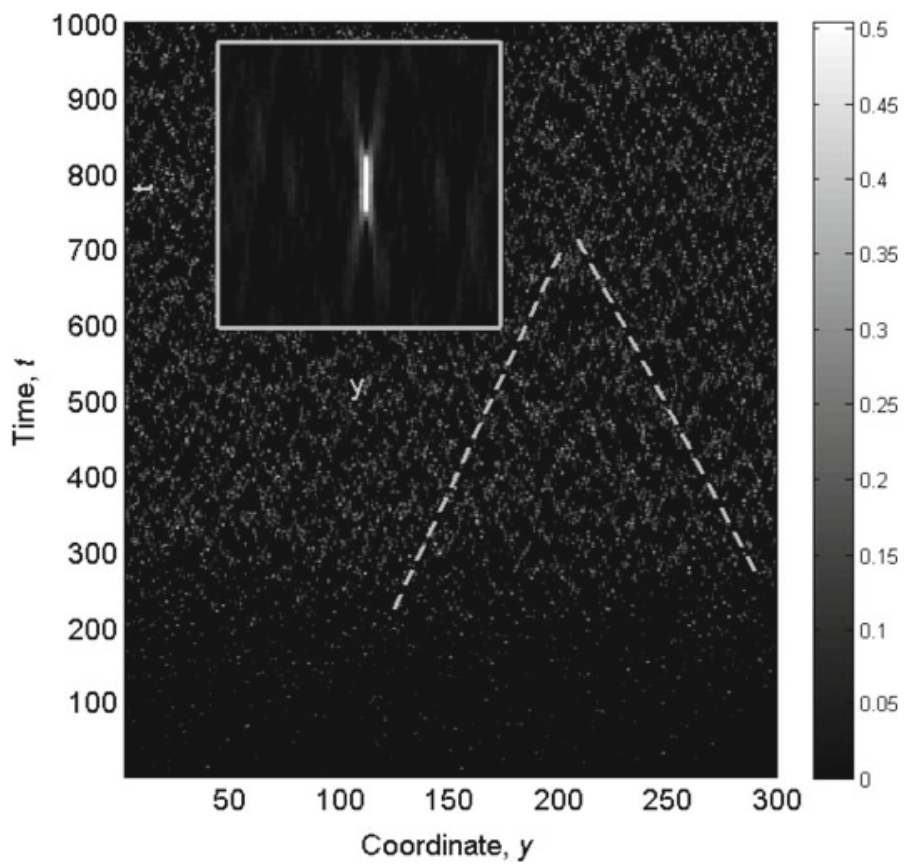

Fig. 5 Spatial-temporal map of jump distribution represented by the $M(t, x)$ matrix, with the intensities indicated by grayscale on the right. The initial stage corresponds to the establishment of a stationary process. Dashed lines correspond to the characteristic velocities of wave propagation (pre- and aftershocks). The inset shows the corresponding $G(t, x)$ correlation function

of the model are the same as described in [12] and above in this Chapter. The initial condition for the further study was selected in the form of a planar front. The resulting spatio-temporal map of jump length distribution along the front is depicted in Fig. 5.

At every step of the numerical procedure, system (1)-(3) is solved and a set of tectonic displacements $\delta X(k)$ (including zero shifts) distributed along the $y$ coordinate is obtained, which represents a row of the $M(t, y)$ matrix at the given time. This procedure is repeated and the entire geological history of the system is recorded in the form of the $M(t, y)$ matrix, which is represented in Fig. 5 by a gray scale map. This spatial-temporal map exhibits a clear initial transition period. A particular scenario is determined by the initial configuration (here, a planar front). As can be seen from Fig. 5, the stationary regime reveals a well-pronounced correlated character. The neighboring regions at the front interact by means of the elastic force $F_{\text {elastic }}=K[X(k+1)+X(k-1)-2 X(k)]$, so that large jumps of one segment induce several jumps in the neighboring segment that propagate as decaying waves in both directions from a strong local "earthquake." Arriving at a certain "weak" segment, i.e., a segment potentially close to a spontaneous break, such waves can initiate this break, inducing a new "tectonic shear" with accompanying waves and 
so on. In other words, each significant event in the system is surrounded by a set of pre- and aftershocks that lead to correlations in the $M(t, y)$ matrix.

The dashed lines in Fig. 5 correspond to the characteristic velocities of wave propagation. Although the velocity of these waves is not universal and varies depending on the constants in Eqs. (1)-(3) their existence is a consequence of the structure of the system under consideration. Physically, the velocity of correlation spreading depends on the composition and strength of rocks, the level of friction forces between blocks, etc.; therefore, each geo-logical region has a certain characteristic velocity, which can be determined from experimental statistics of local secondary shocks accompanying earthquakes. The correlation can be quantitatively described by a spatial-temporal correlation function $G\left(t-t^{\prime}, y-y^{\prime}\right)=\left\langle M(t, y) M\left(t^{\prime}, y^{\prime}\right)\right\rangle$ similar to that depicted in the inset to Fig. 5.

This correlation function was calculated for a particular realization of the $M(t, y)$ matrix over the $t-t_{0}$ time interval (beginning with the time $t_{0}$ found from the termination of the transient process). The gray scale reflects the absolute values of correlations between jumps at the front. The slopped ridges of density $G\left(t-t^{\prime}, y-y^{\prime}\right)$ on both sides of the central maximum correspond to the averaged (typical) velocities of propagation of the interacting events in the given system.

The aim of our investigations is the practical usage of a theoretically justified effect of weak controlled spatially localized impacts on a given system. Previously, we modeled the effect of periodic pulses of variable intensity and preset on/off ratios. If the action is localized in a single $(x, z)$-layer, this layer gradually proceeds forward and pulls the neighboring layers behind to form a protrusion on the $X(k)$ front. Then, increasing deviations $X(k+1)+X(k-1)-2 X(k)$ are sup-pressed by the elastic coupling with neighboring layers. Nevertheless, we succeeded in suggesting a strategy [12] that retained the applicability of the proposed method in a distributed system. For this purpose, each subsequent point of action was shifted over several $(x, z)$ layers along the front so that a small jump would be initiated at a different site of the front, stimulating new neighboring regions. This shift was selected in both the $3 D$-model and its reduced variant.

Figure 6a illustrates such an artificially stimulated process in the same system (and same notation) as in Fig. 5. Here, the clearly distinguished straight lines correspond to periodic impacts regularly shifted along the front, which virtually completely suppress the spontaneous jumps in the systems. Unfortunately, this scenario requires large-scale preliminary works irrespective of whether the probable earthquakes will actually take place. At the same time, the correlations of spontaneous events suggest a constructive idea; it is possible to apply the artificial impacts at the sites of statistically anticipated aftershocks rather than over the entire front, thus only producing a controlled initiation of small jumps at the sites where these jumps are stimulated by intrinsic correlations.

Figure $6 \mathrm{~b}$ shows the distribution of events caused by such a self-consistent action. This pattern appears as more densely filled with jumps as compared to that in Fig. 6a. However, the scales of jump lengths in Fig. 6a, b are also substantially different. The main consequence of this procedure is a sharp drop in the fraction of spontaneous events taking place when the system reaches the level of critical stresses. 

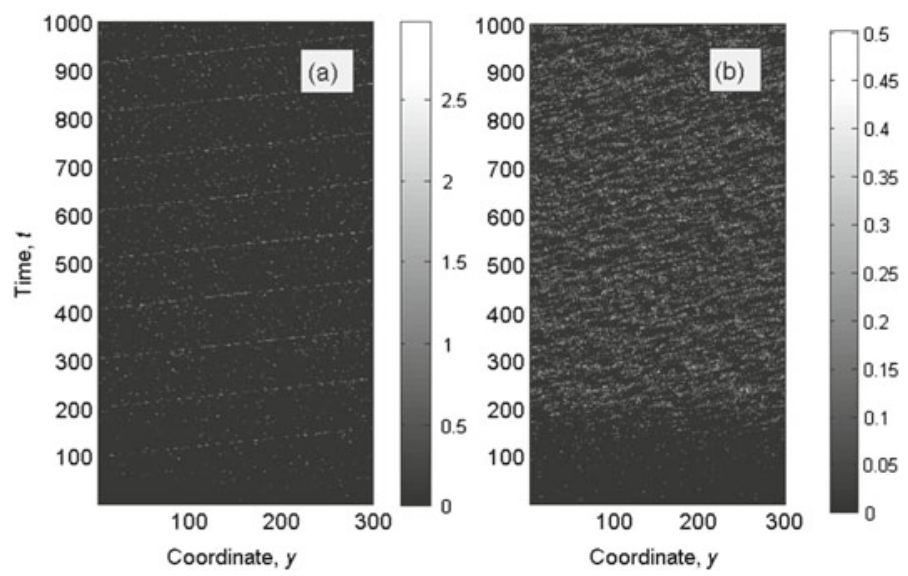

Fig. 6 Spatial-temporal map as in Fig. 5, but in the presence of artificial impacts initiating local jumps of the front segments in the case of a regular shift of the impact site along the entire front and $\mathbf{b}$ adaptive reaction to events selected using the $G(t, x)$ correlation function

Figure 7 shows the temporal variation of the number of such events that were not prevented by the economic adaptive scenario mentioned previously. As can be seen, the relative fraction of critical events is formally large (even reaching unity) only in the initial transient stage, where the events are not yet correlated. However, this stage is an evident artifact of the numerical procedure with a planar initial front (since the initial configuration was a priori not known). In a stationary stage, where the system attains a self-consistent regime, the fraction of spontaneous jumps not prevented by the economic adaptive action falls within $0.1-0.2$. In other words, the

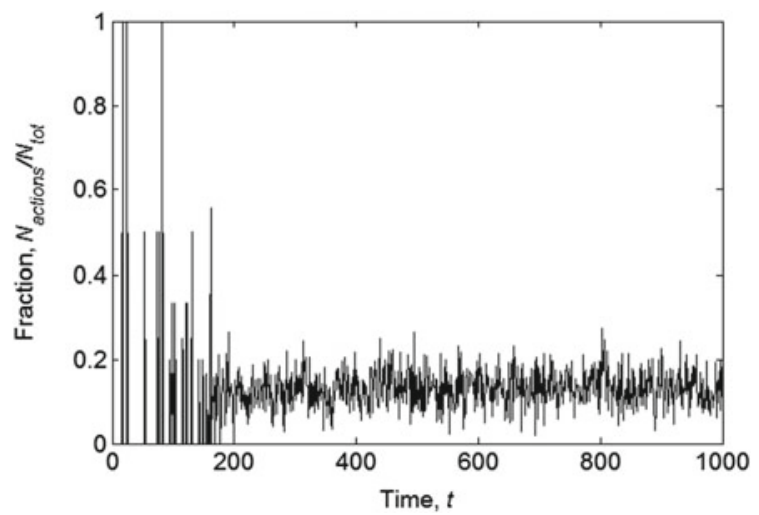

Fig. 7 Temporal variation of the fraction of spontaneous jumps not prevented by the proposed adaptive scenario (mapped in Fig. 6b). In the initial transient stage, the events are not yet correlated and the fraction of spontaneous jumps can be large (reaching unity), while in a stationary stage this fraction falls within $0.1-0.2$ 
economic scenario allows $80-90 \%$ of spontaneous earthquakes in the system to be prevented.

\section{Burridge-Knopoff (BK) Model}

In addition to the above model, we also studied in [14] the well known BurridgeKnopoff (BK) model [15] initially proposed to investigate statistical properties of earthquakes. Numerical studies by Carlson et al. $[16,17]$ have demonstrated that the BK model can reproduce characteristic empirical features of tectonic processes such as the Gutenberg-Richter law for the magnitude distribution of earthquakes, or the Omori law for statistics of aftershocks [16-19], both properties stemming from the so called "self-organized criticality" of this system. It has been intensively used to simulate different aspects of the problem [18-38] and to discuss general properties of earthquakes statistics as well as predictability of earthquakes.

Numerical simulations give evidences that the self-organized criticality and the corresponding fractal attractor of the system is closely related to dynamic structures with "traveling waves" [21], their ordering and specific "phase transitions" [22] controlled by a number of parameters (external driving velocity, springs stiffness, number of blocks, their mutual interaction and so on). It is in particular the dependence of the dynamic properties of the BK model on the spring stiffness, which makes it necessary to introduce the generalization of the friction law proposed in this paper.

The physical reason for the stick-slip instability in the Burridge-Knopoff model is the assumed decrease of the friction force with the sliding velocity [16, 17]. Motion of a single block with this friction law is always unstable which does not correspond to properties of real tribological systems. The real law of rock friction is more complicated [26-31]. In this article, we proceed from more realistic friction laws described in [31, 32]. The main qualitative picture of a realistic law is: (a) approximately logarithmic increase of the static friction force as a function of contact time- the property, found already by Coulomb [39], and (b) a logarithmic dependence of the sliding friction on the sliding velocity. Both properties can be described in the framework of "state dependent" friction laws by introducing additional internal variables describing the state of the contact. Up to now, there were no attempts to study the dynamic and statistic properties of the Burridge-Knopoff model with a state dependent friction law.

In the paper [14], we proposed a modified version of the BK model with a state dependent friction force, reproducing in the simplest way both the velocity weakening friction and the increase of static friction with time when the block is not moving. To validate the model, we studied the stability diagram. It qualitatively reproduces typical diagrams found for almost all tribological systems. The state dependent friction law was also used in $[40,41]$ where an extensive numerical simulation of the one-dimensional spring-block model with such a friction law has been performed and the magnitude distribution and the recurrence-time distribution were studied. 

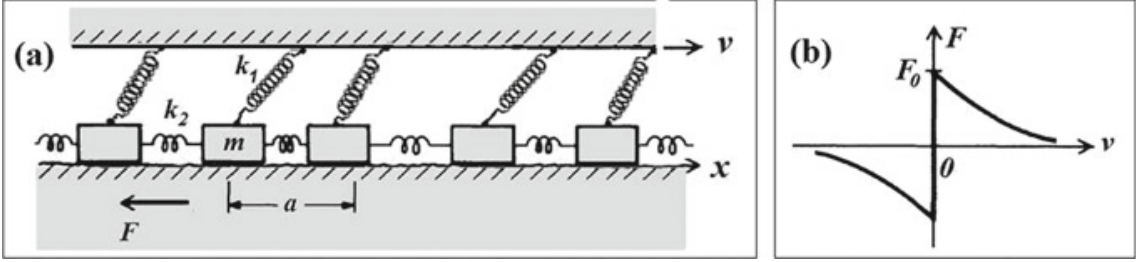

Fig. 8 Burridge-Knopoff (BK) model. Subplot a presents a conceptual scheme of the model. In the simulations we use $m=1$ for the mass and $a=1$ for the distance between the blocks. Subplot b reproduces the original velocity weakening stick-slip friction law used in the Carlson, Langer version of the BK model

Let us start from the original BK model, the conceptual structure of which is depicted in Fig. 8. Blocks of mass $m$ are attached to a moving surface by springs with stiffness $k_{1}$ and are coupled to each other by springs with stiffness $k_{2}$. The moving surface has a velocity $v$, and the blocks are in contact with a rough substrate. The friction force $F$ between the blocks and the rough surface is assumed to depend only on the velocity. Subplot (b) of Fig. 8 reproduces the original velocity dependence of the friction force used in Carlson and Langer version of the BK model. The equations of the BK model can be written in the following form:

$$
m \frac{\partial^{2} u_{j}}{\partial t^{2}}=k_{2}\left(u_{j+1}+u_{j-1}-2 u_{j}\right)+k_{1}\left(v t-u_{j}\right)-F\left(v_{j}\right)
$$

where $v=$ const is the external driving velocity and $v_{j} \equiv \frac{\partial u_{j}}{\partial t}$ is an array of individual block velocities $(j=1, \ldots, N)$.

In the BK model, the sliding friction force is supposed to decreases monotonously from a constant initial value $F_{0}$. It is further supposed that the static friction $F\left(v_{j} \rightarrow\right.$ 0 ) can possess any necessary negative value to prevent back sliding:

$$
F\left(v_{j}\right)=\left\{\begin{array}{cc}
\frac{F_{0}}{1+2 \alpha v_{j} /(1-\sigma)}, & F_{0}=1-\sigma ; \quad \partial u_{j} / \partial t>0 \\
(-\infty, 0] & \partial u_{j} / \partial t=0
\end{array}\right.
$$

Here, according to the original works [16, 17], the parameter $\alpha$ defines a rate of friction decrease when block starts to slide, and $\sigma$ is the acceleration of a block at the instant when slipping begins.

\section{Modified BK Model}

The friction Eq. (10) is a drastic oversimplification of real properties of static and kinetic friction. This equation does not reproduce the correct stability diagram for sliding; with this friction equation the system is always unstable. Experiments show 
that, in most cases of dry friction, sliding stabilizes for either sufficiently large velocities or a sufficiently large stiffness of the system. These friction properties are now well understood and explained in details in the books [31, 32]. Based on friction experiments with rocks, Dieterich [26, 27] has proposed friction equations with internal variables. In his approach, the friction force depends on an additional variable that describes the state of the contact zone. This variable is, in a sense, a "melting parameter." The friction force at non-zero velocity drops down from its initial value due to a "shear melting" effect which may have various physical origins [14-17]. When the motion stops, the surfaces start to form new bonds and the static friction increases with time. These observations become especially important if the model is to be used for describing phenomena with geological characteristic times like earthquakes. Below we follow the ideology of "shear melting effect" and use additional kinetic equations for the friction force.

The dynamics of systems with state dependent friction has been investigated in a number of papers [26-34]. All these studies have been devoted to the simple oneparticle version of the model. In the present paper, we investigate dynamics of the many-body BK model with a state dependent friction. The basic dynamics equations of the model are the same.

$$
m \frac{\partial^{2} u_{j}}{\partial t^{2}}=k_{2}\left(u_{j+1}+u_{j-1}-2 u_{j}\right)-\eta \frac{\partial u_{j}}{\partial t}+k_{1}\left(v t-u_{j}\right)-F_{j}\left[v_{j}(t)\right] .
$$

However, the friction force is not a function of velocity, but is defined by the additional kinetic equation:

$$
\begin{gathered}
\frac{\partial F_{j}\left[v_{j}(t)\right]}{\partial t}=\beta_{1}\left(F_{0}-F_{j}\right)+\beta_{2} v_{j}, \quad \text { with } \beta_{2}<0 v_{j}>0 \\
F\left(v_{j}\right)=-\infty \quad v_{j} \leq 0
\end{gathered}
$$

The parameters in first of these equations $\beta_{1}$ and $\beta_{2}$ have the following physical (and geophysical) meaning. When a block starts to slide with $v_{j}>0$, its friction force monotonously decreases from an initial value $F_{0}$. The general time scale of this process [in relation to other time-scales of the problem, defined by the terms of Eq. (11)] is determined by the first parameter $\beta_{1}$ and an effectiveness of the melting is given by a relation between the absolute value of the negative parameter $\beta_{2}<0$ and $\beta_{1}$.

Static friction $F\left(v_{j} \rightarrow 0\right)$ in second line of Eq. (12) can possess any necessary negative value to prevent back sliding, as in Eq. (10). It is the only nonlinear part of the system, which is found to be enough to create all nontrivial properties of the model. For general applicability to tribological problems Eq. (11) contains also a viscous term $\eta \partial u_{j} / \partial t$. Our calculations show that attractor properties are weakly influenced by the viscous term. Moreover, this influence exists only at sufficiently 
high velocities $v_{j}$. For generality, in the results presented below, we keep small nonzero value $\eta=0.05$.

It should be noted that other forms of the kinetic equations for $\partial F_{j} / \partial t=$ $\beta_{1}\left(F_{0}-F_{j}\right)+\beta_{2} v_{j}$ have been proposed $[14,15]$ leading to qualitatively similar decreasing behavior of the dynamic friction at $v_{j}>0$. Equation (12) is the simplest form. It is also linear. Another reason for this choice is the following. A realistic model must reproduce the correct stick-slip and sliding behavior in appropriate parameter regions. In particular, it must reproduce the correct stability diagram on the plane $\left\{v, k_{1}\right\}$, which typically has an unstable region at small system velocities and small system stiffness $[14,15]$. Note that the original BK model in the form of Eqs. (9) and (10) does not reproduce a stable sliding regime at small sliding velocities and large stiffness. The modified model qualitatively reproduces a typical known stability diagram for sliding friction under various conditions. We have studied the stability diagram by direct dynamic simulation of the BK-system. For this sake, we control the behavior of the complete velocity arrays $\left\{v_{j}(t)\right\}$ for all blocks $j=1, \ldots, N$. To visualize the dynamics of the system, it is convenient to calculate the mean velocity:

$$
\langle v\rangle \equiv\left\langle v_{j}(t)\right\rangle=\frac{\sum_{j=1}^{N} v_{j}(t)}{N} .
$$

Unless otherwise specified, we keep the number of the blocks equal to $N=512$. The main control parameters of the system are the driving velocity $v$ and the stiffness $k_{1}$ of the "springs" connecting the block with the external subsystem. In order to study general properties of the model, let us vary parameters $v$ and $k_{1}$ around the values of order of unity. Typical time dependencies of the mean velocity $\langle v\rangle$ found at free boundary conditions for three representative regions of driving velocity and stiffness constant $v=0.7$ and $k_{1}=0.2 ; v=0.7$ and $k_{1}=2 ; v=2$ and $k_{1}=0.2$ are shown in Fig. 9 subplots $(\mathrm{a}-\mathrm{c})$, respectively.

In regions (b) and (c), the fluctuations of the velocity disappear after a transient period, whereas in the stick-slip region (a) the velocity fluctuates continuously. The dynamics of the friction force

$$
\left\langle F_{\text {fric }}\right\rangle=\left\langle F_{j}(t)+\eta v_{j}(t)\right\rangle
$$

is shown in the same plot.

Performing the simulation for various combinations of parameters, we have found the desired stability diagram, shown in Fig. 10, for the modified BK model in the plane $\left\{v, k_{1}\right\}$. The line separates the regions of sliding and stick-slip motion. Higher intensity of gray color corresponds to bigger oscillations of friction force. As discussed above, this characterizes the amplitude of the force variation at different spring stiffness $k_{1}$ and driving velocity $v$. If the interaction between the blocks is relatively weak, these strong variations of friction can lead, in principle, to a state in which different blocks of the chain can simultaneously be found in moving and stacked states. This observation is important for studying of the model further. Below we 


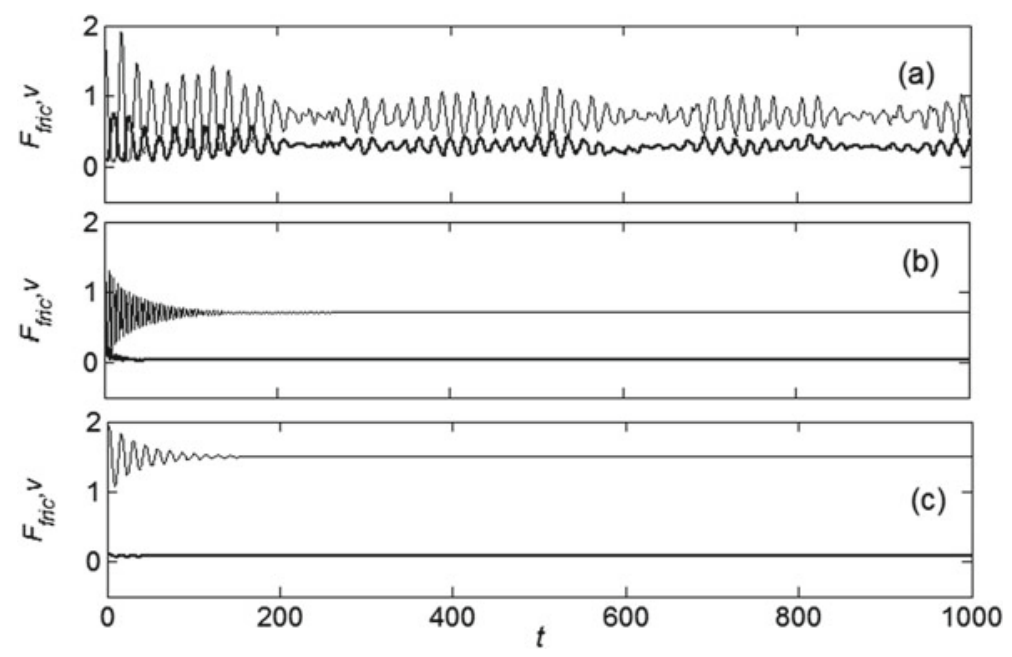

Fig. 9 Time dependencies of mean velocity $\langle v\rangle$ and friction force $\left\langle F_{\text {fric }}\right\rangle=\langle F+\eta v\rangle$ found for three representative regions of the driving velocity and stiffness constant $v=0.7$ and $k_{1}=0.2$; $v=0.7$ and $k_{1}=2 ; v=2$ and $k_{1}=0.2$ shown in (a)-(c) subplots, respectively. In the sliding regions $\mathbf{b}$ and $\mathbf{c}$ the fluctuations of the velocity disappear after a transient period of initial oscillations, whereas in stick-slip region (a) both mean velocity and a correlated friction force remain perpetually variant

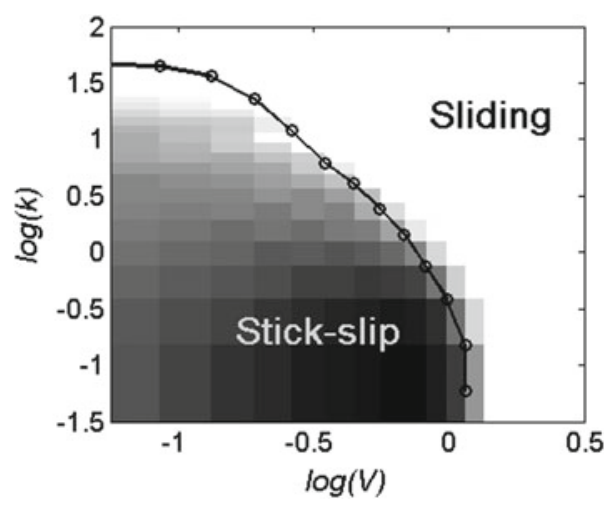

Fig. 10 Stability diagram for the modified BK model (MBK). The line separates the region of stable sliding from that of variable stick-slip motion. The grayscale map represents the standard deviation value of the complete friction force $\left\langle F_{\text {fric }}\right\rangle=\left\langle F_{j}\left[v_{j}(t)\right]+\eta v_{j}(t)\right\rangle$ in stationary stickslip regime. It characterizes the amplitude of the force variation at different spring constants $k$ and driving velocities $v$. Higher gray color saturation corresponds to bigger friction force oscillations (with black color corresponding to maximum of standard deviation equal to 0.72 ) 
study the dynamic and statistical properties of the new model with the previously described realistic state dependent friction law. We will refer to the set of Eqs. (12), (13) as the modified BK model (MBK).

\section{Attractor Properties, Wave State and Phase Transition in a 1-Dimensional Model}

The equations of motion in (12) are a discrete representation of a nonlinear wave equation. During the last few decades various nonlinear wave equations have been widely studied, starting from the very early implementation of the numerical simulations [35]. In particular, in the context of dynamic "thermalization" it has been shown that $N$ interacting segments of the nonlinear chain form a collective attractor with energy transfer performed by nonlinear excitations [36, 37, 42, 43]. Depending on the total energy and/or on the strength of the interaction between the blocks $k_{2}$, the chain can form (nearly) uniform or strongly non-uniform structures and phase patterns.

Analogous behavior should be expected in the MBK model as well. It is obvious that instantaneously moving blocks must be involved with stationary ones in the overall motion. This causes a "detachment" wave, propagating along the chain. Such solitary waves were found and studied in the original BK model [15], and the analogous process exists in the MBK model. There are two possible types of traveling waves: (a) after a transition time all the blocks are provoked to move simultaneously, (b) in steady-state, some of the blocks can be found instantaneously motionless while others move. One would expect that the response of the chain will depend on the strength of the interaction between the blocks. This has been studied for the original BK model in [17], and a specific "phase transition" between correlated and uncorrelated behavior has been found.

Let us study this problem for the MBK model. Figure 11 presents typical waves of local block velocities in a "stationary" regime for parameter values $k_{2}=4, k_{1}=$ 1 and $v=0.2$. Non-zero velocities are shown here by the spatiotemporal mesh surface. Traveling and mutually scattering waves are clearly visible. Let us draw the attention to the areas of intensive local spikes appearing as result of mutually scattering of waves. These spikes have displacement amplitudes $\left|u_{j}\right| \gg\left\langle u_{j}\right\rangle$ and velocities $\left|v_{j}\right| \gg\left\langle v_{j}\right\rangle$ much higher than the mean values. They are relatively rare (the area occupied by these events is much smaller than the total space-time area), and in applications for geodynamics they should be treated as "earthquake events".

To characterize the difference of the correlated and uncorrelated behavior, an order parameter has been introduced in Ref. [22]. If we define a value $h_{j}$ in block number $j$ by the condition:

$$
h_{j}= \begin{cases}1 & \partial \mathrm{u}_{j} / \partial t \neq 0 \text { (the block is moving) } \\ 0 & \partial \mathrm{u}_{j} / \partial t=0\end{cases}
$$




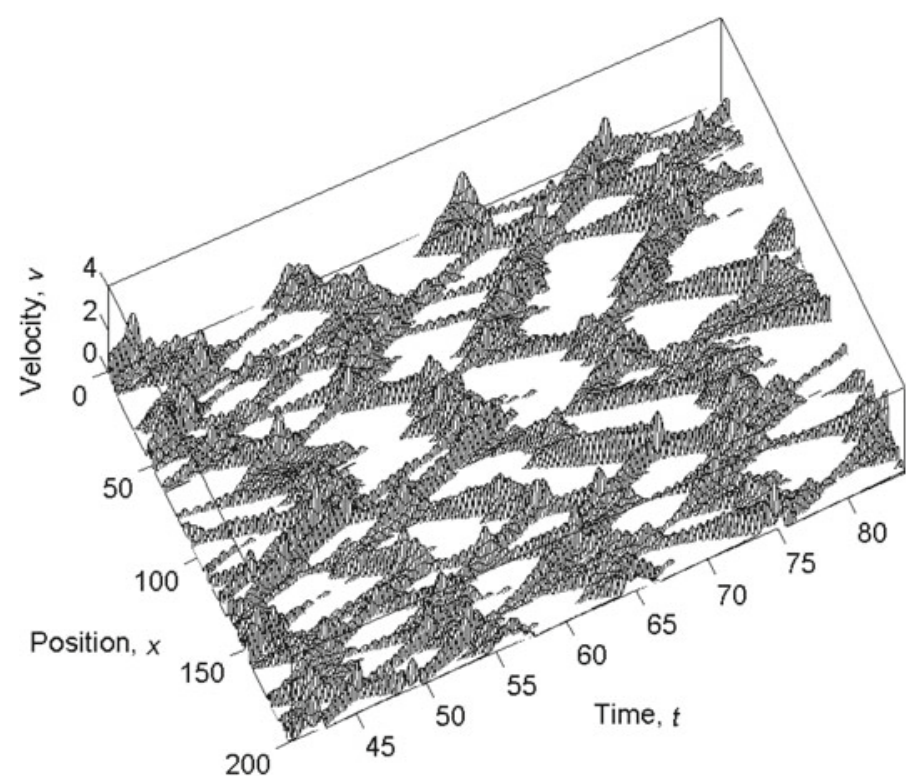

Fig. 11 Typical waves of the local velocity (of each discrete block of the system) for the developed regime in a one-dimensional system. The non-zero velocities are shown by the spatiotemporal mesh surface. The areas of intensive local events due to mutual scattering of waves are clearly visible. Parameter values: $v=0.7, k_{1}=0.2, k_{2}=4, N=512, \beta_{1}=1, \beta_{2}=25, \eta=0.05, m=$ 1.

then the local density of the order parameter $H_{j}^{*}$ can be written as $H_{j}^{*}=h_{j}\left(h_{j+1}+\right.$ $\left.h_{j-1}\right)$. This function takes the unit value $H_{j}^{*}=h_{j}\left(h_{j+1}+h_{j-1}\right)=1$ if block $j$ is moving and exactly one of its nearest neighbors is also moving. Further, $H_{j}^{*}=$ $h_{j}\left(h_{j+1}+h_{j-1}\right)=2$ when both nearest neighbors of the moving block are in motion. All other cases yield $H_{j}^{*}=0$.

Our observations with the MBK model show that even for blocks at vanishing inter-block interaction $k_{2} \rightarrow 0$ (when the motion of neighboring blocks is almost uncorrelated), the fraction of configurations with moving sets of neighboring blocks is still relatively high. The combination $H_{j}^{*}=h_{j}\left(h_{j+1}+h_{j-1}\right)$ does not vanish in such an uncorrelated system. It is therefore convenient to construct another simple combination:

$$
H_{j}=h_{j} h_{j+1} h_{j-1}= \begin{cases}1 \text { all } 3 \text { blocks } j, j+1, j-1 \text { in contact are moving } \\ 0 & \text { otherwise }\end{cases}
$$

This yields $H_{j}=h_{j} h_{j+1} h_{j-1}=0$ zero in all cases except when both neighboring blocks of a central sliding block are also in motion. This combination can be used as an "order parameter". 

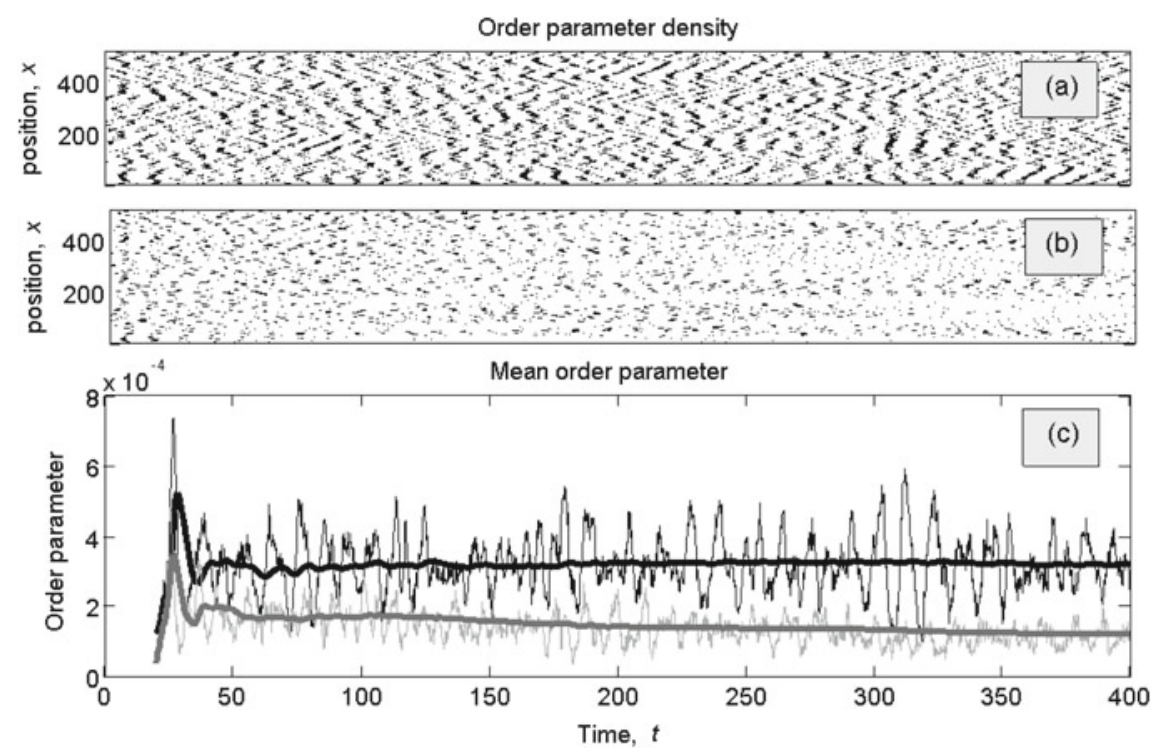

Fig. 12 Order parameter density and its time dependence in two different regimes. Subplots a and b show the grayscale maps of the order parameter density depending on time and space for strong and weak coupling between the neighboring blocks ( $k_{2}=4$ and $k_{2}=1.2$ respectively). Time evolution of the ensemble-averaged order parameter for (a) and (b) cases are presented in the subplot (c) by black and gray tick lines respectively. The time averages for these values are shown by the bold lines of corresponding color. Parameter values: $v=0.7, k_{1}=0.2, k_{2}=4, N=512, \beta_{1}=$ $1, \beta_{2}=25, \eta=0.05, m=1$.

Figure 12 shows the order parameter density and time dependence of its ensemble average $\left\langle H_{j}(t)\right\rangle$ in two different regimes. Subplots (a) and (b) show grayscale maps of the order parameter density depending on time and space for strong and weak coupling between neighboring blocks $\left(k_{2}=4\right.$ and $k_{2}=1.2$ respectively). Time dependence of the mean value $\left\langle H_{j}(t)\right\rangle$ is presented in the subplot (c) with black and gray thick lines for the (a) and (b) cases, respectively.

To extract integral quantitative information about the steady ordered and disordered states let us calculate the time evolution of the ensemble-averaged order parameter:

$$
H(t) \equiv\left\langle H_{j}(t)\right\rangle=\frac{1}{t} \int_{0}^{t}\left\langle H_{j}(t)\right\rangle d t .
$$

These time-averaged values for the cases (a) and (b) are shown in the subplot (c) by the bold lines overlapping respective thick curves $\left\langle H_{j}(t)\right\rangle$. The long-time stationary asymptote $\left\langle H_{j}(t)\right\rangle \rightarrow$ const can characterize the behavior of the system in an integral manner. 


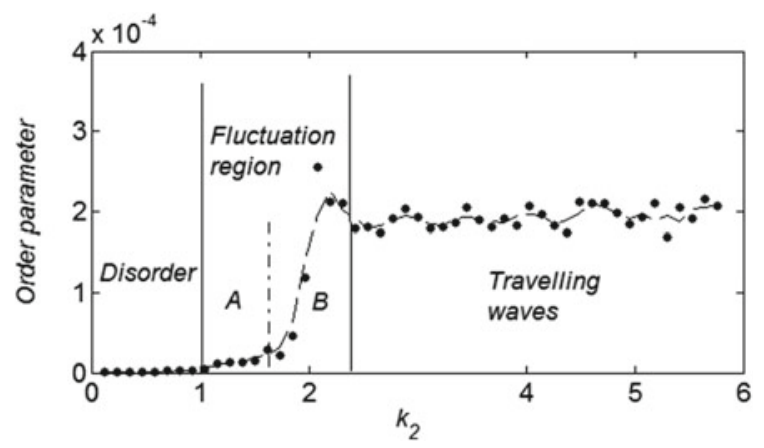

Fig. 13 Phase transition from correlated to uncorrelated motion of blocks in a one-dimensional system. The order parameter tends to a constant asymptote at high mutual interaction $k_{2} \gg 1$ and vanishes below transition point $k_{2}=k_{2}^{\text {critical }} \simeq 1$. Other parameters are the same as in Fig. 5. The ordered state corresponds to the nearly regular waves (seen clearly in Fig. 11). Two intermediate fluctuation regions $\mathrm{A}$ and $\mathrm{B}$ correspond to states with short-range and long-range correlated nonlinear excitations, respectively

The dependence of the integrated order parameter on the stiffness $k_{2}$, showing a transition from correlated to uncorrelated block motion in a one-dimensional system, is shown in Fig. 13. Two limiting cases can be identified: (a) the order parameter tends to a constant non-zero asymptote $\left\langle H_{j}(t)\right\rangle \rightarrow$ const $\neq 0$ at strong interaction $k_{2} \gg 1$, and (b) it vanishes $\left\langle H_{j}(t)\right\rangle \rightarrow 0$ below the transition point $k_{2} \approx 1$.

The ordered state corresponds to the nearly regular waves presented in Fig. 12. We distinguish two fluctuation regions $\mathrm{A}$ and $\mathrm{B}$ at intermediate interaction. These regions can be characterized by two clearly different order parameter mean values in Fig. 13. One can verify further that they also differ dynamically and correspond to states with short range- and long-range correlated nonlinear excitations, respectively. This intermediate behavior may characterize the physically important features of the model under consideration.

\section{Study of the 2-Dimensional Model}

The real contact of two surfaces is two-dimensional. Let us generalize the MBK model for the $2 \mathrm{D}$ case. The generalized model is very similar to the 1-dimensional model, but incorporates a 2D array of blocks connected by elastic springs in both directions. All other components of the MBK remain unchanged.

The system of equations of motion takes the form:

$$
\begin{aligned}
m \frac{\partial^{2} u_{j, n}}{\partial t^{2}}= & k_{2}\left(u_{j+1, n}+u_{j-1, n}+u_{j, n+1}+u_{j, n-1}-4 u_{j, n}\right) \\
& -\eta \frac{\partial u_{j, n}}{\partial t}+k_{1}\left(v t-u_{j, n}\right)+F\left(v_{j, n}\right)
\end{aligned}
$$




$$
\begin{gathered}
\frac{\partial F_{j, n}\left(v_{j, n}(t)\right)}{\partial t}=\beta_{1}\left(F_{0}-F_{j, n}\right)+\beta_{2} v_{j, n} ; \text { with } \beta_{2}<0, v_{j, n}>0, \\
F\left(v_{j, n}\right)=-\infty \quad v_{j, n} \leq 0
\end{gathered}
$$

where: $j=1, \ldots, N_{x}$ and $n=1, \ldots, N_{y} . N_{x}$ and $N_{y}$ are the numbers of elements in the $x$ - and $y$-directions. It is possible to repeat all the simulations of the previous section, reproduce all the results presented in Figs. 9, 10, 11, 12 and 13, and show that these properties are quite common between the 1D and 2D MBK models.

In particular, one can obtain a wave state in two dimensions. The only difficulty appears in a visualization of the results, depending on 3 coordinates $\{x, y ; t\}$. As an example, Fig. 14 presents the mentioned wave state in the two-dimensional model. In contrast to the 2-dimensional $\{x ; t\}$ space-time maps with a complete history of events in Figs. 11 and 12 the subplots (a) and (b) now represent instantaneous
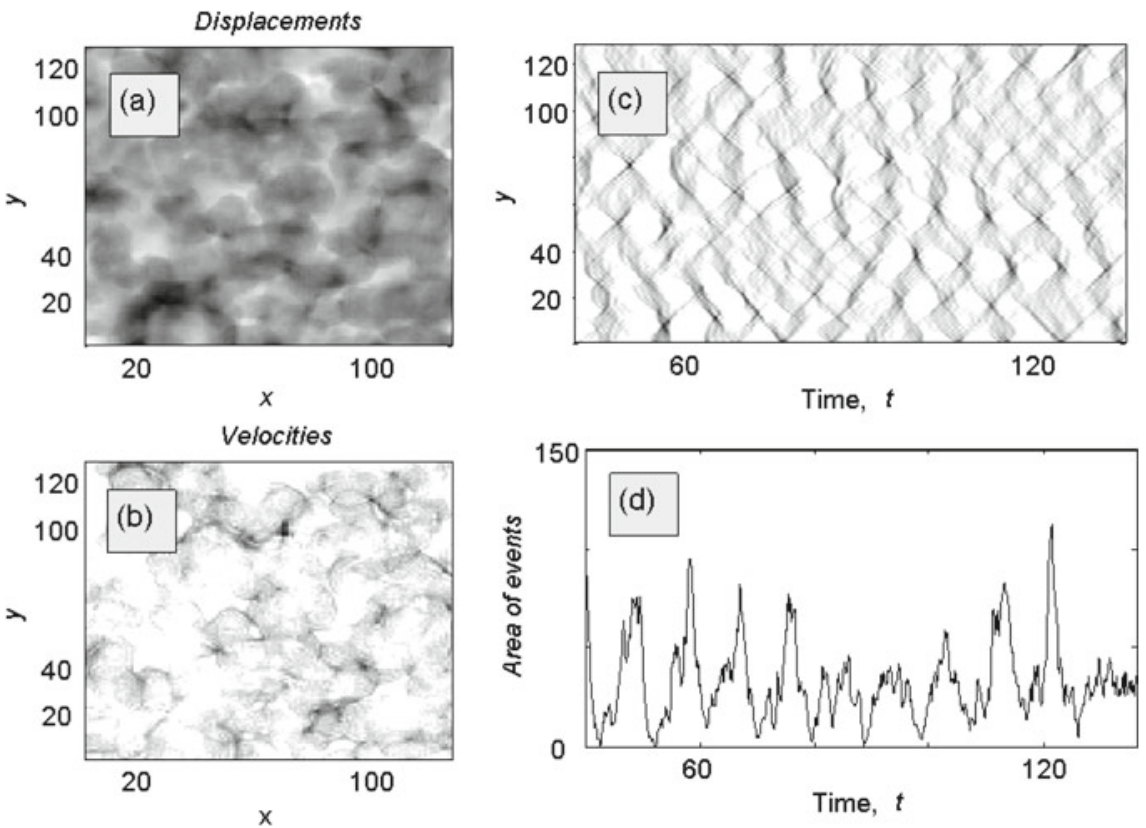

Fig. 14 Wave state in the two-dimensional model. Subplots $\mathbf{a}$ and $\mathbf{b}$ represent snapshots of the instantaneous densities of the local displacements $u=u(x, y ; t)$ and velocities $v=v(x, y ; t) \equiv$ $\partial u / \partial t$, respectively. The darkest color corresponds to the value 3 indimensionless units and white corresponds to zero. Mutual scattering manifests itself in high sharp peaks of the "events" reproduced here by the dark gray spots of the corresponding densities. A time-space representation of this process is shown in (c) by the cross-section of the $\{x, y ; t\}$-space along one of the $x=$ const planes. Ensemble averaged area of the events corresponding to the same process is plotted in subplot (d). The number of blocks is equal to $N_{x} \times N_{y}=128 \times 128$ and other parameters are the same as in Fig. 12 
snapshots of the density distribution (for the local displacements $u=u(x, y ; t)$ and velocities $v=v(x, y ; t) \equiv \partial u / \partial t$ respectively). However, direct observation of the time-dependent numerical simulations shows that the waves (clearly visible in the Fig. 14) are moving $2 D$ fronts of the excitations. These fronts conserve their shape for relatively long periods of time. Their mutual scattering manifests itself in high and sharp peaks of the "events". Corresponding spikes are well reproduced by dark gray spots of the density distributions in subplots (a) and (b) of Fig. 14.

Some record of the process which has led to the presented instantaneous distributions is shown in subplot (c) by means of a cross-section of the $\{x, y ; t\}$-space along one of the planes where $x=$ const. One can also calculate an ensemble averaged area of the events corresponding to the same process. This is plotted in Fig. 14 subplot (d). There is obvious correlation between the subplots (c) and (d); however, the correlation is not complete. In reality, the total area of events includes a summation over all the planes $j=1, \ldots, N_{x}$ and involves plenty of impacts from other $x=$ const planes which are invisible in the subplot (c).

Nevertheless, the correlation is easily seen and looks much stronger than one would expect in such case. The traveling waves influence the motion of blocks neighboring in both directions $\{x, y\}$. Therefore, there is a certain correlation between the densities along all 3 time-space coordinates $\{x, y ; t\}$. In order to reproduce this in a static picture, we present a 3-dimensional density distribution for the exact same process in Fig. 15. This figure combines the density of "events" (black volumes) with the grayscale maps discreetly depicted for certain sub-planes: $t=$ const, $x=$ const and $y=$ const.

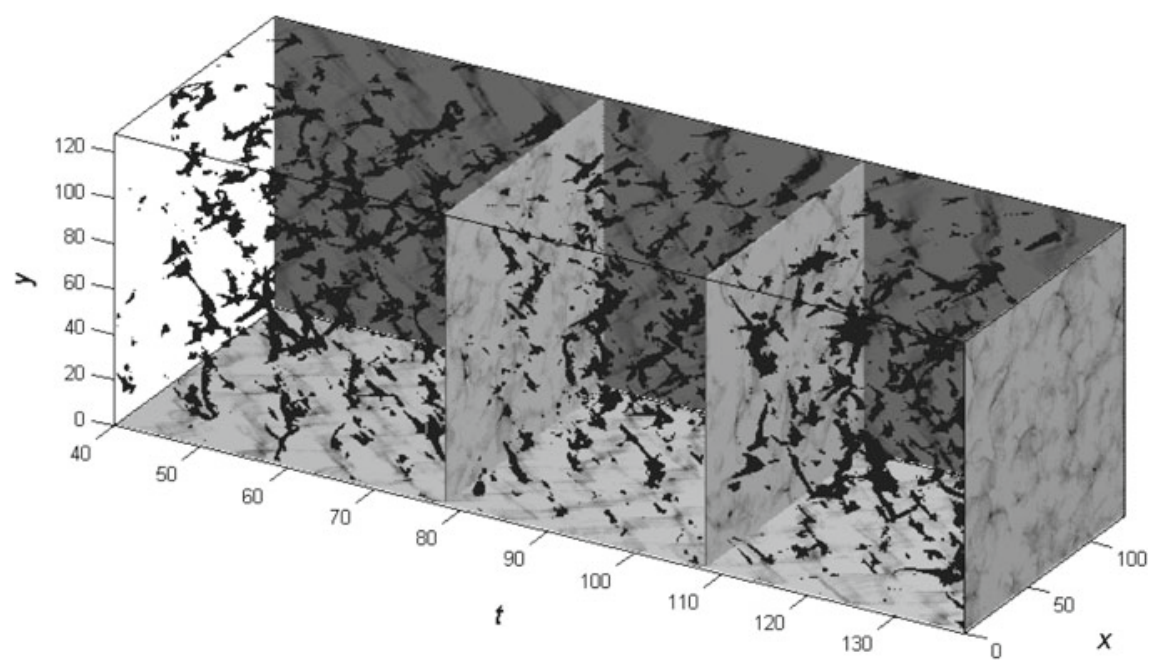

Fig. 15 Density of events (black volumes) in a two-dimensional system $N_{x} \times N_{y}=128 \times 128$ at the same parameters as in Figs. 12 and 13. Grayscale maps (with the same gradations as in previous figure) for some representative planes $t=$ const, $x=$ const and $y=$ const are added to compare with Fig. 13 


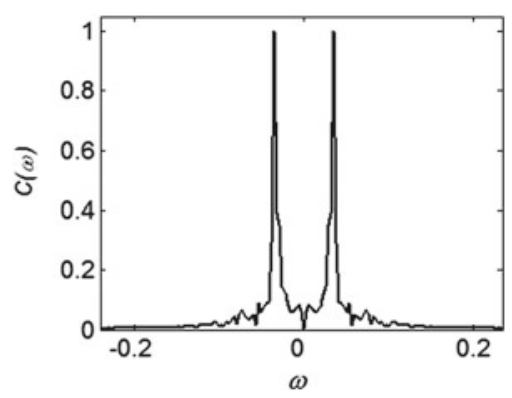

Fig. 16 Fourier transform $C(\omega)$ of the two-time correlation function $G\left(t_{2}-t_{1}\right)$

There is a noticeable periodicity in the total area of events in Figs. 14 and 15. To illustrate this, we have calculated a two-time correlation function $G\left(t_{2}-t_{1}\right)$ for the total area and taken its Fourier transform $G_{\omega}$. The resulting Fourier transform is presented in Fig. 16. It smoothes random impacts from the time-fluctuations and possesses obvious maxima corresponding to a characteristic frequency of the total area oscillation. The frequency of these large-scale collective oscillations is determined by the parameters of the problem. According to our numerical experiments, the characteristic frequency can be varied mainly by changing the driving velocity, external springs and constants $\beta_{1,2}$ in the equation $\partial F_{j, n}\left[v_{j, n}(t)\right] / \partial t=$ $\beta_{1}\left(F_{0}-F_{j, n}\right)+\beta_{2} v_{j, n}$. It is important to stress here that the observed behavior corresponds to a global attractor of the dynamic system (19) and (20). This means that it corresponds to the stationary asymptotic behavior of the system, independent of initial conditions.

In all the cases presented in previously mentioned figures, we omitted initial time intervals corresponding to the transient period. This part of the evolution can be different and depends on the initial conditions. We have checked this by starting the simulations from almost uniform distribution of the low velocities, from small displacements, from intensive random noise, or by changing open boundary conditions (normally used here) to the periodic ones, and so on. In all cases, the system quickly suppresses unfavorable fluctuations, vents to an attracting "large river" common for all the transient scenarios, and slowly attracts along the "river" to the stationary scenario. This kind of evolution is mathematically typical for many nonlinear systems [38] and the MBK model is no exception.

The attractor manifests itself in a stationary distribution $\rho=\rho\left\{u, F_{\text {fric }}, v, \ldots\right\}$ of the dynamic variables in a phase space. Figure $17 \mathrm{a}, \mathrm{b}$ present its projections in two different sub-planes of the phase-space: planes $\{u, v\}$ and $\left\{F_{\text {fric }}, v\right\}$, respectively. By accumulating the density $\rho=\rho\{u, \ldots, v, \ldots\}$ onto a grayscale map in subspace $\{u, v\}$, one can see the correlation between the time-depending fluctuations of displacements $u(x, y ; t)$ and velocities $v(x, y ; t)$.

It can be shown that the dense central part of the distribution is mainly due to multiple but weak oscillations of small amplitude ("phonons") and basal areas of the traveling waves. The widely extended depopulated gentle slopes with low density 

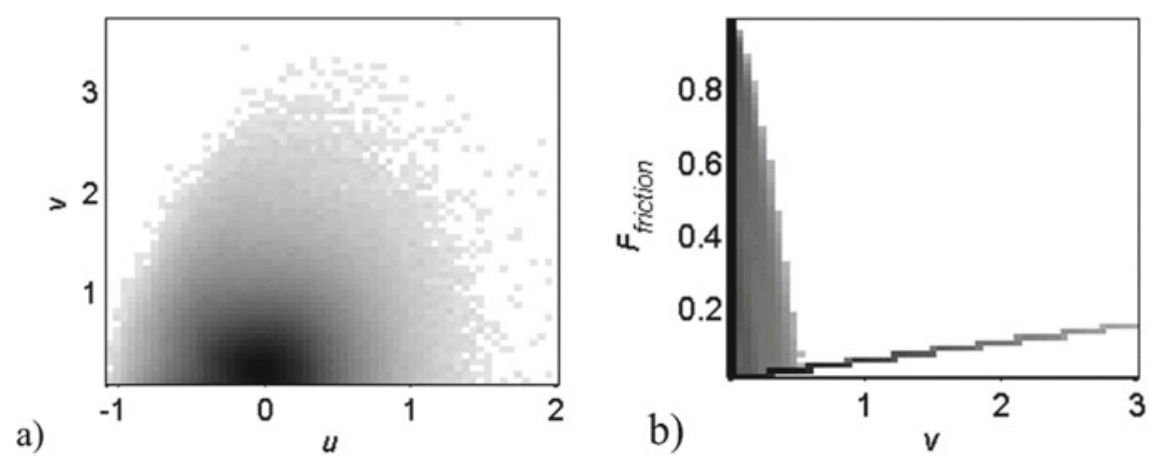

Fig. 17 a Grayscale map of the density of dissipative attractor states projected onto the phase sub-space $\{u, v\}$. b The same attractor as in Fig. 17 a projected onto the sub-space $\left\{v, F_{\text {friction }}\right\}$, where $\left\langle F_{\text {friction }}\right\rangle=\left\langle F_{j}\left[v_{j}(t)\right]+\eta v_{j}(t)\right\rangle$

$\rho=\rho\{u, \ldots, v, \ldots\}$ in peripheral regions of the $\{u, v\}$ surface are produced by rare intensive "events" [which cause high spikes of displacements $u(x, y ; t)$ and velocities $v(x, y ; t)]$. In other words, statistical study of the rare "earthquake events" in the frame of the MBK model is equivalent to the study of the outer periphery of its dissipative attractor.

Another projection of the attractor onto the $\left\{F_{\text {fric }}, v\right\}$ subspace shown in the Fig. 17b can be used to control correct correspondence between statistically preferable behavior of the dynamically complete friction force $F_{\text {fric }}=F\left[v_{n, j}(t)\right]+$ $\eta v_{n, j}(t)$ with the "naive", physically expected dependence $F=F_{f r i c}(v)$. Finally, let us return again to the discussion of Fig. 17a. The inherent structure of the attractor with extended gentle slopes of the density $\rho=\rho\{u, \ldots, v, \ldots\}$ corresponding to rare intensive "events" gives a simple and clear image for the origination of scaling asymptotic distributions. To obtain these, one must cut off the outer areas along both the displacement and velocity coordinates. Corresponding asymptotic distributions obtained after such a cut-off are reproduced in subplots (a) and (b) of Fig. 18, respectively. The inserts to the figures illustrate the power-law nature of both distributions.

Comparing the models one can conclude that the standard BK model utilizes a velocity weakening friction force to reproduce the correct statistical behavior of "events". In contrast, the MBK model includes an additional phenomenological equation, subsequently providing a self-consistent dynamic description of the velocity depending friction force. This modification has at least two advantages: it realistically generates the velocity weakening friction force of the moving blocks and provides growth of static friction for the locked blocks. The model was studied for different driving velocities and driving springs elastic constants. It was possible to build a stability diagram for the transition between smooth sliding and stick-slip behavior, which was in good qualitative agreement with what is expected experimentally. Further numerical study under a broad range of parameters proved that the MBK model reproduces all important features of the standard BK model (traveling 

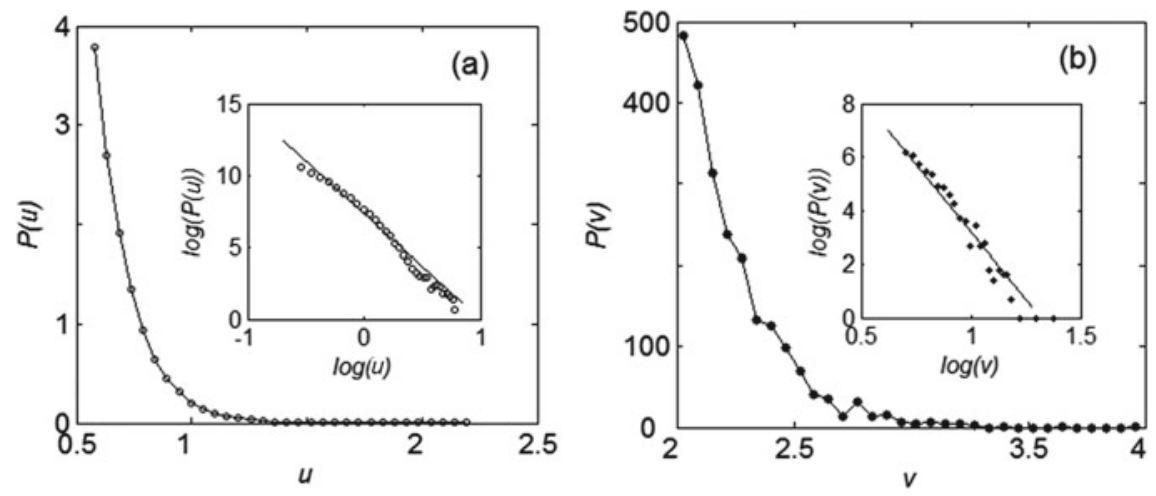

Fig. 18 Scaling relations in the model. To get scaling behavior one must cut-off the external lightgray regions with power-law density of states from the attractor in Fig. 17. Physically it corresponds to a selection of the rare but intensive "events", which is compatible with the ideology of the empirical Gutenberg-Richter law

waves, attractor properties of dynamic equations in one- and two-dimensional cases, and so on).

\section{References}

1. Gutenberg B, Richter CF (1944) Frequency of earthquakes in California. Bull Seismol Soc Am 34(4):185-188

2. Bak P, Christensen K, Danon L, Scanlon T (2002) Unified scaling law for earthquakes. Phys Rev Lett 88:178501

3. Omori F, Coll J (1895) On the aftershocks of earthquakes. J Coll Sci Imperial Univ Tokyo 7:111-120

4. Bak P, Tang Ch, Wiesenfeld K (1987) Self-organized criticality: an explanation of the $1 / \mathrm{f}$ noise. Phys Rev Lett 59(4):381-384

5. Carlson JM, Langer JS (1989) Properties of earthquakes generated by fault dynamics. Phys Rev Lett 62:2632

6. Ruzhich VV, Smekalin OP, Shilko EV, Psakhie SG (2002) About nature of "slow waves" and initiation of displacements at fault regions. In: Proceedings of international conference "new challenges in mesomechanics", Aalborg University, Denmark, Aug 26-30, 2002, vol 1, pp 311-318

7. Ruzhich VV, Truskov VA, Chernykh EN, Smekalin OP (1999) Russ Geol Geophys 40:356

8. Psakhie SG, Shilko EV, Astafurov SV (2004) Peculiarities of the mechanical response of heterogeneous materials with highly deformable interfaces. Tech Phys Lett 30:237-239

9. Psakhie SG, Ruzhich VV, Shilko EV, Popov VL, Dimaki AV, Astafurov SV, Lopatin VV (2005). Influence of the state of interfaces on the character of local displacements in fault-block and interfacial media. Tech Phys Lett 31(8):712-715. https://link.springer.com/article/10.1134/1. 2035374

10. Ruzhich VV, Psakhie SG, Bornyakov SA, Smekalin OP, Shilko EV, Chernykh EN, Chechelnitsky VV, Astafurov SV (2002) Investigation of influence of vibroimpulse excitations on regime of displacements in seismically active fault regions. Phys Mesomech 5(5-6):85 
11. Fermi E, Pasta J, Ulam S, Tsingou M (1955) Studies of non linear problems. Los Alamos Report Laboratory of the University of California, LA-1940

12. Filippov AÉ, Popov VL, Psakhie SG, Ruzhich VV, Shilko EV (2006) Converting displacement dynamics into creep in block media. Tech Phys Lett 32:545-549

13. Filippov AÉ, Popov VL, Psakhie SG (2008) Correlated impacts optimizing the transformation of block medium dynamics into creep regime. Tech Phys Lett 34(8):689-692

14. Filippov AÉ, Popov VL (2010) Modified Burridge-Knopoff model with state dependent friction. Tribol Int 43:1392-1399

15. Burridge R, Knopoff L (1967) Model and theoretical seismicity. Bull Seismol Soc Am 57(3):341-371

16. Bruce SE, Carlson JM, Langer JS (1992) Patterns of seismic activity preceding large earthquakes. J Geophys Res Solid Earth 97(B1):479

17. Carlson JM, Langer JS, Shaw BE (1994) Dynamics of earthquake faults. Rev Mod Phys 66(2):657-670

18. Saito T, Matsukawa H (2007) Size dependence of the Burridge-Knopoff model. In: Journal of physics: conference series, International conference on science of friction, 9-13 Sept 2007, Irago, Aichi, Japan, vol 89, p 012016

19. Mandelbrot BB (1982) The fractal geometry of nature. Freeman and Co, San Francisco

20. Gutenberg B, Richter CF (1954) Seismicity of the earth and associated phenomena. Princeton University Press, Princeton (New Jersey)

21. Muratov CB (1999) Traveling wave solutions in the Burridge-Knopoff model. Phys Rev E 59:3847

22. Huisman BAH, Fasolino A (2005) Transition to strictly solitary motion in the Burridge-Knopoff model of multicontact friction. Phys Rev E 72(1Pt2):016107

23. Clancy I, Corcoran D (2005) Criticality in the Burridge-Knopoff model. Phys Rev E 71(4Pt2):046124

24. Clancy I, Corcoran D (2006) Burridge-Knopoff model: exploration of dynamic phases. Phys Rev E 73(4Pt2):046115

25. Mori T, Kawamura H (2008) Simulation study of earthquakes based on the two-dimensional Burridge-Knopoff model with long-range interactions. Phys Rev E 77(5Pt1):051123

26. Dieterich JH (1974) Earthquake mechanisms and modeling. Annu Rev Earth Planet Sci 2:275301

27. Dieterich JH (1978) Time-dependent friction and the mechanics of stick-slip. Pure Appl Geophys 116:790-806

28. Rice JR (1983) Earthquake aftereffects and triggered seismic phenomena. Pure Appl Geophys 121:187-219

29. Gu JC, Rice JR, Ruina AL, Tse ST (1984) Slip motion and stability of a single degree of freedom elastic system with rate and state dependent friction. J Mech Phys Solids 32(3):167-196

30. Marone C (1998) Laboratory-derived friction laws and their application to seismic faulting. Annu Rev Earth Planet Sci 26:643-696

31. Persson BNJ (2000) Sliding friction, physical properties and applications. Springer, Berlin

32. Popov VL (2010) Contact mechanics and friction. Foundations and applications. Springer, Berlin

33. Popov VL (2000) A theory of the transition from static to kinetic friction in boundary lubrication layers. Solid State Commun 115(7):369-373

34. Filippov AE, Klafter J, Urbakh M (2004) Friction through dynamical formation and rupture of molecular bonds. Phys Rev Lett 92(13):135503

35. Fermi E, Pasta J, Ulam S, Tsingou M (1993) In: Mattis DC (ed) The many-body problem: an encyclopedia of exactly solved models in one dimension. World Scientific Publishing Co Pte Ltd., Singapore

36. Braun OM, Hu B, Filippov A, Zeltser A (1998) Traffic jams and hysteresis in driven onedimensional systems. Phys Rev E Stat Phys Plasmas Fluids Relat Interdisc Top 58(2):1311

37. Fillipov A, Hu B, Li B, Zeltser A (1998) Energy transport between two attractors connected by a Fermi-Pasta-Ulam chain. J Phys A Gen Phys 31(38):7719 
38. Filippov AE (1994) Mimicry of phase transitions and the large-river effect. JETP Lett 60(2):141

39. Popova E, Popov VL (2015) The research works of Coulomb and Amontons and generalized laws of friction. Friction 3(2):183-190

40. Persson BNJ, Popov VL (2000) On the origin of the transition from slip to stick. Solid State Commun 114(5):261-266

41. Ohmura A, Kawamura H (2007) Rate-and state-dependent friction law and statistical properties of earthquakes. EPL (Europhys Lett) 77(6):69001

42. Ford J (1992) The Fermi-Pasta-Ulam problem: paradox turns discovery. Phys Rep 213(5):271310

43. Lichtenberg AJ, Lieberman MA (1992) Regular and chaotic dynamics. Springer, New York

Open Access This chapter is licensed under the terms of the Creative Commons Attribution 4.0 International License (http://creativecommons.org/licenses/by/4.0/), which permits use, sharing, adaptation, distribution and reproduction in any medium or format, as long as you give appropriate credit to the original author(s) and the source, provide a link to the Creative Commons license and indicate if changes were made.

The images or other third party material in this chapter are included in the chapter's Creative Commons license, unless indicated otherwise in a credit line to the material. If material is not included in the chapter's Creative Commons license and your intended use is not permitted by statutory regulation or exceeds the permitted use, you will need to obtain permission directly from the copyright holder. 